CASE REPORT

\title{
Unusual cardiac tumour with perivascular myoid differentiation: a case report
}

\author{
A Orlandi, A Ferlosio, A Ciucci, A Pellegrino, L G Spagnoli
}

J Clin Pathol 2004;57:1338-1340. doi: 10.1136/icp.2004.020081

A previously healthy 70 year old woman was admitted for fatigue and dyspnoea on exertion and cough. A two dimensional echocardiography revealed a mass in the right atrium, which obstructed filling and infiltrated the cardiac chamber wall. Postsurgical histological examination revealed an unusual tumour with prevalent myoid glomangiopericytoma-type and haemangiopericytoma-like patterns. Mitosis and necrosis were absent. A computed tomography scan excluded the presence of metastasis to distant organs or, conversely, metastatic involvement of the heart. Therefore, a diagnosis of tumour with perivascular myoid differentiation was made. This new entity, recently described in soft tissues, can easily recur. Its recognition helps to differentiate from metastasis and other primitive cardiac tumours sharing some morphological features but a different clinical behaviour, with consequent improvement to the management of patient care.

W e present the case of a previously healthy 70 year old woman, who was admitted for fatigue and dyspnoea on exertion and cough. Heart failure occurred and a functional examination was made. The $x$ ray showed only an accentuation of pulmonary patterns. An electrocardiogram showed sinus rhythm. A two dimensional echocardiography revealed a mass in the right atrium, which obstructed filling and infiltrated the cardiac chamber wall (fig 1). Her clinical history and computed tomography scans excluded a metastatic origin of the tumour or, conversely, the presence of metastasis to distant organs. The tumour was surgically removed along with the right atrium roof, which was replaced by an autologous pericardial patch. A cardiopulmonary bypass was required.

\section{PATHOLOGY}

On gross examination, the mass was ovoid in shape, apparently capsulated, of brown colour with a lacerated, bleeding surface, and measured $4 \times 5.5 \times 3.5 \mathrm{~cm}$. A histological examination of paraffin wax embedded sections showed an unusual tumour with prevalent glomangiopericytoma-type and haemangiopericytoma-like patterns (fig 2A-D). ${ }^{1}$ In some areas, glomangiopericytoma-type gaping cavernous spaces, myoid nodules, and perivascular hyalinisation were prevalent. In other areas, the prominent anatomising vessels were lined by a single row of factor VIII, CD34 positive endothelial cells (fig 2E). This "staghorn" branching pattern differed from that of classic haemangiopericytoma in that tumour cells of solid areas showed a diffuse muscle specific actin and $\alpha$ smooth muscle actin positive myoid or myofibroblastic phenotype (fig 2F). ${ }^{2}$ Desmin positive cells were also present. Myxoid degeneration, as demonstrated by
Alcian-Pas staining, and the "clear" aspect of scattered cells were also characteristic of myofibroblastic or myoid differentiation. Mitosis and necrosis were absent. A focal chronic inflammatory infiltrate was also present. Immunohistochemical keratin, S-100, and epithelial membrane antigen negativity of tumour cells excluded other cardiac primitive and secondary tumours. ${ }^{2}$

\section{DISCUSSION}

We describe here an unusual tumour with perivascular myoid differentiation. The histological aspect of this tumour, largely composed of mainly round undifferentiated to epithelioid cells around prominent, thin walled "staghorn" vessels, was suggestive of haemangiopericytoma. In contrast, the CD34 negative and the diffuse $\alpha$ smooth muscle actin positive phenotype of the tumour cells excluded that diagnosis. ${ }^{2}$ In addition, glomangiopericytomatous areas were also detected. Therefore, the diagnosis of a primitive tumour of the heart with myoid perivascular differentiation was made. This definition was recently reported for those soft tissue tumours with both perivascular myoid and myopericytic or myofibroblastic differentiation, including cases of myofibromatosis in adults, glomangiopericytoma, and myopericytoma. ${ }^{13}$ Perivascular myoid tumours arise over a wide age range, are classically located in subcutaneous tissues of the extremities, ${ }^{13}$ are often asymptomatic for a long period of time, and are sometimes congenital. Recurrence is possible after surgery. ${ }^{1}$ Recently, a wider anatomical distribution has been recognised. ${ }^{4}$ To our knowledge, this is the first case of a tumour with perivascular myoid differentiation of the heart described in the literature. This might be because of its intrinsic rarity and the fact that until now it was included among cases of haemangioma or haemangiopericytoma.

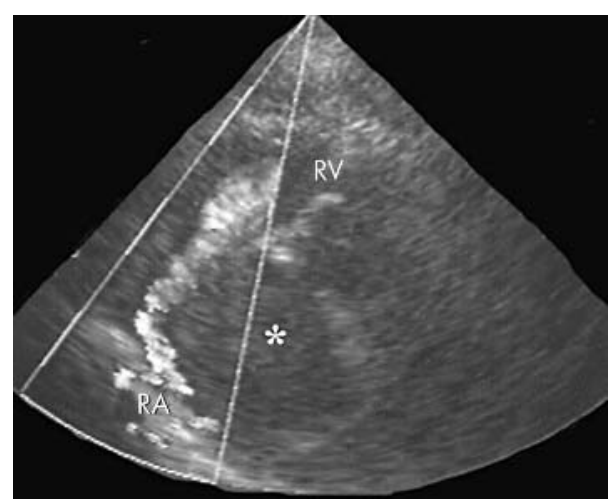

Figure 1 Two dimensional echocardiography showing a right cardiac mass, which obstructed atrial filling and infiltrated the wall (RV, right ventricle; RA, right atrium; asterisk, cardiac mass). 

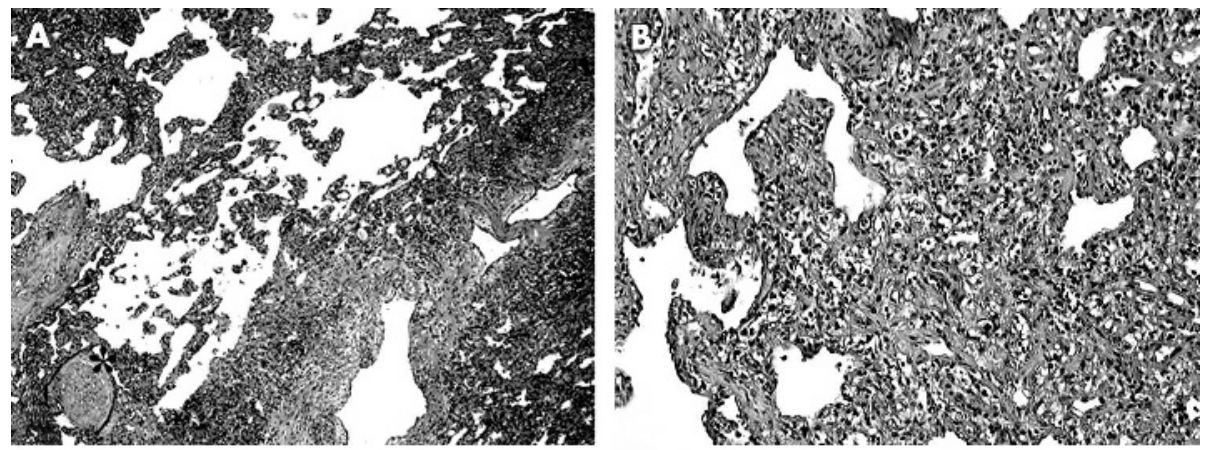

Figure 2 (A) Glomangiopericytomatype and (B) haemangiopericytoma-like patterns of perivascular myoid tumour of the heart. (C) Well defined edge of the fumour at the site of atrial origin. (D) At higher magnification, there is perivascular distribution of mainly round cells, sometimes with a clear aspect of cytoplasm.

(E) Immunohistochemical features of a "staghorn" branching pattern lined by a single row of CD34 positive cells, whereas (F) $\alpha$ smooth muscle actin positive staining demonstrates the myofibroblastic differentiation of the
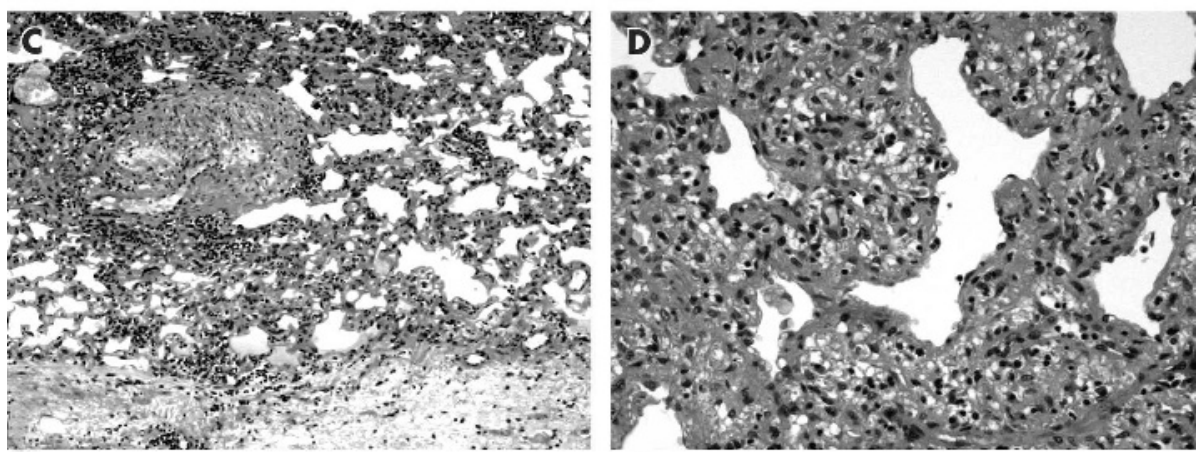
tumour cells. The asterisk in (A)
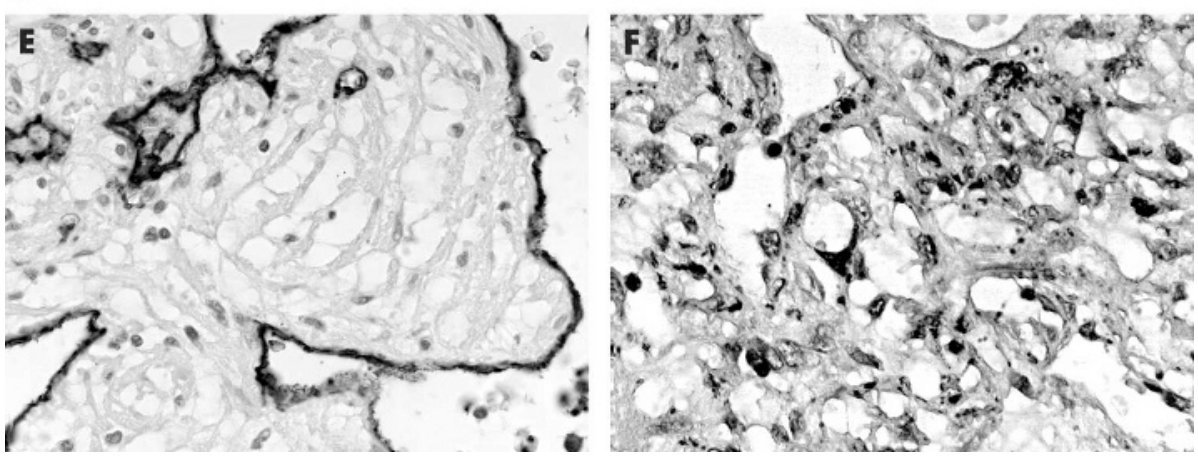
indicates a myoid nodule.

"To our knowledge, this is the first case of a tumour with perivascular myoid differentiation of the heart described in the literature"

With regard to the biological behaviour of perivascular myoid tumours, they have the tendency to recur, especially in adults. ${ }^{1}$ McMenamin and Fletcher described five cases of malignant myopericytoma, expanding the spectrum of soft tissue myoid perivascular tumours. ${ }^{5}$ They shared morphological and phenotypic similarities with their benign counterparts, although a careful examination demonstrated some worrisome features, such as high cellularity, frequent mitoses, and necrosis. ${ }^{5}$ In our case, the absence of metastasis at diagnosis and after six months of follow up supports the histologically benign appearance of the tumour. In any case, differential diagnosis should also include other tumours with a variable differentiation towards myoid cells/pericytes, such

\section{Take home message}

- Perivascular myoid tumour is unusual in the heart and must be distinguished from other cardiac tumours sharing some morphological features but with a different clinical behaviour as solitary myofibroma, epithelioid haemangioendothelioma, glomus tumour, and vascular leiomyoma, in addition to some malignant tumours, such as leiomyosarcoma, malignant peripheral nerve sheath tumour, monophasic synovial sarcoma, myxofibrosarcoma, and malignant fibrous histiocytoma. ${ }^{25}$

In conclusion, it is important to take into account a possible cardiac localisation of a myoid perivascular tumour, to achieve the correct differential diagnosis of primitive and secondary cardiac tumours sharing some morphological features but with a different clinical behaviour.

\section{Authors' affiliations}

A Orlandi, A Ferlosio, A Ciucci, L G Spagnoli, Anatomic Pathology, Tor Vergata University, Via Montpellier 1, Rome 00133, Italy

A Pellegrino, Cardiovascular Surgery, Tor Vergata University

Correspondence to: Professor A Orlandi, Anatomic Pathology Institute, Department of Biopathology, Tor Vergata University of Rome, Via Montpellier 1, Rome, Italy; orlandi@uniroma2.it

Accepted for publication 20 June 2004

\section{REFERENCES}

1 Granter SR, Badizadegan K, Fletcher CD. Myofibromatosis in adults, glomangiopericytoma, and myopericytoma: a spectrum of tumors showing perivascular myoid differentiation. Am J Surg Pathol 1998;22:513-25. 
2 Burke AP, Virmani R. Tumors of the heart and great vessels. In: Atlas of tumor pathology, Fascicle 16, 3rd ed. Washington, DC: Armed Forced Institute of Pathology, 1996:80-6.

3 Kutzner H. Perivascular myoma: a new concept for "myofibroblastic" tumors with perivascular myoid differentiation. Verh Dtsch Ges Pathol 1998:82:301-8.
4 Li XQ. Hisaoka M, Morio T, et al. Intranasal pericytic tumors (glomus tumor and sinonasal hemangiopericytoma-like tumor): report of two cases with review of the literature. Pathol Int 2003;53:303-8.

5 McMenamin ME, Fletcher CD. Malignant myopericytoma: expanding the spectrum of tumours with myopericytic differentiation. Histopathology 2002;41:450-60. 